\title{
Activity of Neurons in Human Temporal Cortex during Identification and Memory for Names and Words
}

\author{
George A. Ojemann and Julie Schoenfield-McNeill \\ Department of Neurological Surgery, University of Washington, Seattle, Washington, 98195
}

Extracellular recordings of human temporal cortical neuronal activity during identification and memory for object names or words were obtained from 31 neurons at 18 sites in 12 left language dominant patients undergoing left (10) or right (2) awake craniotomy for epilepsy under local anesthesia. Frequency of activity during identification was compared with perceptual controls, that during the encoding phase of recent memory to identification of the same material. Statistically significant changes in one or more temporal epoch $(p<0.005)$ of one or more comparisons were present for 27 of the 31 neurons in either hemisphere. Few neurons changed activity in the same direction for both words and names. The instruction to retain an item in recent memory changed activity in most neurons from that during identification of the same material, although the items presented were identical and overtly identified in each task. Any individual neuron usually changed activity in one

The importance of human temporal lobe for memory was initially established by the observation of amnesia after bilateral lesions there (Scoville and Milner, 1957). That deficit is most evident in recent memory for specific items and declarative episodic memory but not long-term memory, very brief working memory, or memory for procedures (Shimamura, 1989). Subsequent studies demonstrated lateralized material-specific recent memory deficits: verbal after left temporal resections and visuospatial after right temporal resections (Milner, 1971). These deficits are usually related to damage to medial temporal structures, especially hippocampus (Squire and Zola-Morgan, 1991). However, there is also evidence indicating a role for lateral temporal neocortex in recent memory, derived from effects of lesions (Milner, 1967; Ojemann and Dodrill, 1987) and electrical stimulation mapping (Ojemann, 1978, 1983; Fried et al., 1982; Ojemann and Dodrill, 1985; Perrine et al., 1994).

Neurosurgical operations in which the patient is awake for part of the procedure provide an opportunity to record activity of neurons in human temporal neocortex (Ojemann et al., 1998). Those recordings have shown changes in neuronal activity during measures of recent memory for names (Ojemann et al., 1988; Haglund et al., 1994) and words (Weber and Ojemann, 1995;

Received Jan. 11, 1999; revised April 21, 1999; accepted April 21, 1999.

This work was supported by National Institutes of Health Grant NS 36527 and a McDonnell-Pew Cognitive Neuroscience grant. We thank E. Lettich for assistance during intraoperative recording and preparation of the figures, Drs. D. Corina and E. Fetz for reviewing earlier versions of this manuscript, and Sheela Forler for her preparation of the many revisions of this manuscript.

Correspondence should be addressed to George A. Ojemann, University of Washington, Department of Neurological Surgery, 1959 N.E. Pacific Street, Box 356470, Seattle, WA 98195-6470.

Copyright (C) 1999 Society for Neuroscience $\quad 0270-6474 / 99 / 195674-09 \$ 05.00 / 0$ direction for only one task. There are separate, widely distributed neural networks for identification or recent memory for each type of material. The majority of nearby neurons recorded through the same extracellular microelectrode were related to the networks for different tasks.

The temporal characteristics of these changes were also investigated; $31 \%$ of the changes were "phasic": temporally related to presentation or response to the item. Most of the remaining neuron changes were sustained throughout a task, often for several minutes. These task-specific sustained changes may reflect effects of thalamo-cortical attentional systems. Individual neurons had both sustained and phasic changes to different tasks.

Key words: human; neuron; temporal; cortex; memory; language

Ojemann and Schoenfield-McNeill, 1998). Both increases and decreases in activity have been observed with these recent memory measures. However, each of those studies was conducted in a separate series of patients. Thus, it is not known whether the same neuron changes activity with recent memory for all types of verbal material (words and names) or whether memory for each type of material depends on separate neural networks. The present study was designed to address this question and to provide more detail on the changes in human temporal cortical neuronal activity during recent memory for words or names.

A portion of this study has been published previously in abstract form (Ojemann and Schoenfield-McNeill, 1997).

\section{MATERIALS AND METHODS}

Subjects. Subjects were 12 patients undergoing temporal resections for medically refractory epilepsy using a standard technique in which the patient is awake for a portion of the operation under local anesthesia, so that physiological guides unpreturbed by anesthesia can be used to plan the operation (Ojemann, 1995). Eight subjects were female. Ages ranged from 22 to 57 (mean of 37); preoperative verbal IQs ranged from 75 to 113 (mean of 91). Two female patients underwent right temporal resections and the remainder left. This microelectrode study and methods for obtaining informed consent from each patient were annually approved by the University of Washington Institutional Review Board. All patients were left dominant for language based on preoperative intracarotid amobarbital perfusion testing (Wada and Rassmussen, 1960).

Recording technique. The microelectrode study was performed after completion of the physiological recording and stimulation needed to plan the resection, when the patient had been awake under local anesthesia of $0.5 \%$ lidocaine and $0.25 \%$ marcaine for at least $1 \mathrm{hr}$. Recording used one or more commercial tungsten microelectrode(s) back-loaded through a translucent $1 \mathrm{~cm}$ diameter footplate into one or more hydraulic microdrive. The footplate was used to dampen cortical pulsations. Care was taken to be sure that pial vessels were not blanched by footplate pressure. Recording was confined to temporal neocortex, which was to be subse- 


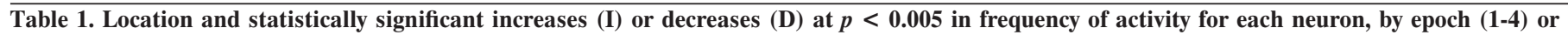
summed over epochs (O)

\begin{tabular}{|c|c|c|c|c|c|c|c|c|c|c|c|}
\hline \multirow[b]{2}{*}{$\begin{array}{l}\text { Patient } \\
\text { Number }\end{array}$} & \multirow[b]{2}{*}{$\begin{array}{l}\text { Age/ } \\
\text { Gender }\end{array}$} & \multirow[b]{2}{*}{$\begin{array}{l}\text { Electrode/ } \\
\text { Neuron }\end{array}$} & \multirow[b]{2}{*}{$\begin{array}{l}\text { Location/ } \\
\text { Depth }\end{array}$} & \multicolumn{3}{|c|}{ Identification } & \multicolumn{5}{|c|}{ Memory encoding (M) } \\
\hline & & & & $\begin{array}{l}\text { Name ID } \\
\text { vs Percep- } \\
\text { tual Con- } \\
\text { trol-N }\end{array}$ & diff & $\begin{array}{l}\text { Word ID } \\
\text { vs Percep- } \\
\text { tual Con- } \\
\text { trol-R }\end{array}$ & $\begin{array}{l}\text { Name } M \text { vs } \\
\text { Name ID }\end{array}$ & diff & $\begin{array}{l}\text { Word M vs } \\
\text { Word ID }\end{array}$ & $\begin{array}{l}\text { Name M } \\
\text { vs Percep- } \\
\text { tual Con- } \\
\text { trol-N }\end{array}$ & $\begin{array}{l}\text { Word M } \\
\text { vs Percep- } \\
\text { tual Con- } \\
\text { trol-R }\end{array}$ \\
\hline \multicolumn{12}{|c|}{ Right middle temporal gyrus-superior half } \\
\hline \multirow[t]{3}{*}{1} & $36 / \mathrm{F}$ & $3 / 1$ & $44 / 3$ & $\mathrm{I} 2-4,0$ & - & $\mathrm{D} 2$ & - & $<0$ & $\mathrm{I} 2$ & $\mathrm{I} 2,0$ & - \\
\hline & & $3 / 2$ & $44 / 3$ & - & - & - & - & - & - & - & - \\
\hline & & $3 / 3$ & $44 / 3$ & - & - & - & - & $>1$ & D23 & - & - \\
\hline \multirow[t]{4}{*}{2} & $41 / \mathrm{F}$ & $1 / 1$ & $36 / 2$ & $\mathrm{I} 23$ & $>2-40$ & D4 & D3 & - & $\mathrm{I} 40$ & - & $\mathrm{I} 3$ \\
\hline & & $3 / 2$ & $51 / 2$ & - & $<1240$ & - & - & - & D10 & - & - \\
\hline & & $4 / 1$ & $51 / 1.5$ & D30 & $>30$ & - & $\mathrm{I} 240$ & $>240$ & I0 & - & I0 \\
\hline & & $4 / 2$ & $51 / 1.5$ & - & - & - & - & - & - & - & - \\
\hline \multicolumn{12}{|c|}{ Left middle temporal gyrus-superior half } \\
\hline \multirow[t]{2}{*}{3} & $23 / \mathrm{F}$ & $1 / 1$ & $35 / 1$ & D20 & - & D2 & - & - & - & D1 & - \\
\hline & & $1 / 2$ & $35 / 1$ & - & $<1-30$ & - & I2-40 & - & - & $\mathrm{I} 3$ & - \\
\hline \multirow[t]{3}{*}{4} & 45/M & $3 / 1$ & $38 / .5$ & - & - & - & - & $>30$ & D0 & - & D30 \\
\hline & & $3 / 2$ & $38 / .5$ & D4 & $>2$ & $\mathrm{D} 2$ & $\mathrm{I} 1,4$ & - & $\mathrm{I} 2$ & I1 & - \\
\hline & & $3 / 3$ & $38 / .5$ & - & - & I1-30 & D3 & - & $\mathrm{D} 2$ & $\mathrm{D} 1,0$ & $\mathrm{I} 3,0$ \\
\hline 5 & $29 / \mathrm{F}$ & $1 / 1$ & $40 / 4$ & $\mathrm{I} 30$ & $>3$ & - & D1 & - & - & - & - \\
\hline \multirow[t]{2}{*}{6} & $57 / \mathrm{F}$ & $3 / 1$ & $34 / 2.5$ & - & - & - & D1340 & - & - & - & - \\
\hline & & $3 / 2$ & $34 / 2.5$ & - & - & - & D1-40 & - & - & D0 & - \\
\hline 7 & $29 / \mathrm{F}$ & $3 / 1$ & $40 / 3$ & D2 & $>1-40$ & - & D30 & $>0$ & $\mathrm{I} 2-40$ & D20 & $\mathrm{I} 2-40$ \\
\hline \multicolumn{12}{|c|}{ Left middle temporal gyrus_-inferior half } \\
\hline \multirow[t]{2}{*}{8} & $37 / \mathrm{F}$ & $3 / 1$ & $42 / 2$ & D20 & $<2$ & $\mathrm{I} 2$ & - & - & - & D2 & - \\
\hline & & $3 / 3$ & $42 / 2$ & - & $<0$ & I0 & - & - & - & - & - \\
\hline \multirow[t]{3}{*}{9} & $45 / \mathrm{M}$ & $1 / 1$ & $34 / 1$ & - & $>1-40$ & - & D20 & - & $\mathrm{I} 230$ & - & $\mathrm{I} 20$ \\
\hline & & $4 / 1$ & $44 / 1$ & - & - & I1230 & - & - & - & $\mathrm{D} 2$ & $\mathrm{I} 0$ \\
\hline & & $3 / 1$ & $44.5 / 1$ & I0 & $>0$ & - & D2 & $<23$ & I0 & - & I20 \\
\hline \multirow[t]{5}{*}{10} & 36/M & $1 / 1$ & $26 / 1$ & $\mathrm{I} 20$ & - & - & - & - & - & - & - \\
\hline & & $1 / 2$ & $26 / 1$ & - & $<1230$ & - & - & $<0$ & - & - & - \\
\hline & & $3 / 1$ & $46 /<1$ & - & - & - & - & - & - & - & - \\
\hline & & $4 / 1$ & $46 / 3$ & - & - & - & - & - & D30 & - & D1340 \\
\hline & & $4 / 2$ & $46 / 3$ & D3 & $<23$ & - & $\mathrm{I} 2$ & - & - & - & - \\
\hline \multicolumn{12}{|c|}{ Left inferior temporal gyrus } \\
\hline \multirow[t]{2}{*}{11} & $22 / \mathrm{F}$ & $3 / 1$ & $45 / 2$ & D240 & $<2340$ & $\mathrm{I} 2$ & I2340 & - & D0 & - & - \\
\hline & & $3 / 2$ & $45 / 2$ & - & $>0$ & - & - & - & D1 & - & - \\
\hline \multirow[t]{3}{*}{12} & $46 / \mathrm{M}$ & $4 / 1$ & $40 / 2.5$ & D20 & $<2$ & - & - & - & $\mathrm{I} 30$ & - & $\mathrm{I} 30$ \\
\hline & & $4 / 2$ & $40 / 2.5$ & - & $>2$ & - & - & - & I0 & - & I0 \\
\hline & & $4 / 3$ & $40 / 2.5$ & - & - & D1 & - & - & - & - & - \\
\hline
\end{tabular}

quently resected as part of the surgical treatment for the patient's epilepsy, in anterior middle temporal gyrus or superior lateral margin of inferior temporal gyrus, from 26 to 51 (mean 40 ) $\mathrm{mm}$ from the temporal tip, at depths of $0.5-4 \mathrm{~mm}$ (Table 1). Other than avoiding recording from neurons with evidence of injury or epileptiform burst activity (Calvin et al., 1973), recordings are from an unselected random sample of neurons in this cortex. Activity from each microelectrode, the patient's responses, and markers for presentation of each item of the behavioral measures were recorded on frequency modulation (FM) tape for later analysis. System frequency response for microelectrode channels was $100-6500 \mathrm{~Hz}$.

Behavioral measures. Once stable recordings were obtained, the patient engaged in a series of tasks designed to measure identification and recent memory for both words and names, along with perceptual controls for each type of material. This study was an extension of our earlier studies of recent memory for words or names in separate patient series; thus, the same sets of behavioral measures were used as in the earlier studies. Each task was presented as a block of trials, with the order of blocks for names or words varied between subjects. Individual items in each task were presented as slides from a Kodak (Eastman Kodak, Rochester, NY) Ektagraphic 260 projector, with each item shown for $4 \mathrm{sec}$.
The measure of identification and recent memory for names (Haglund et al., 1994) used a set of slides of monochromatic object pictures with a red stripe at varying angles across the object. In the eight trials of the identification measure, the patient named the objects aloud as they appeared. Each of the eight trials of the perceptual control for names consisted of two slides. The first slide in each pair was one of the slides used in the identification task but presented with the instruction to view the slide silently. The second slide in each pair had only a red stripe on it. The patient indicated with an overt "yes" or "no" whether the angle of the line on the second slide matched that on the immediately preceding first slide of the pair. Line angle matching has been related to function of the nondominant hemisphere (Benton et al., 1975). Each of the six trials of the measure of recent memory for names consisted of five slides. The first slide in each trial was the same as one of those used in the identification measure and as first slides of the perceptual measure, but with the instruction not only to name the pictured object aloud but also to remember that name. The second, third, and fourth slides in each trial were pictures of other objects, with the patient trained to name them aloud. The fifth slide had the word "recall" on it. In response to this slide, the patient had been trained to state aloud the name of the object 
pictured on the first slide of that trial. Thus, each trial of this task represents a Brown-Peterson measure of recent memory for names, with the first slide requiring encoding, the second, third, and fourth distractors during which the memory must be stored, and the fifth, retrieval.

The measure of identification and recent memory for words (Weber and Ojemann, 1995) used slides of 20 words of common concrete nouns. In the word identification task, eight of these words were presented with the instruction to read the word aloud. In the perceptual control for words, the same words were presented backward, with the instruction to view them silently. Each of the six trials of the recent memory for words also consisted of five slides, with the first slide having one of the same words used in the identification task but with the instruction to not only read it aloud but also to remember it. The next three slides of each trial were pictures of other words with the patient trained to only read them aloud. These were followed by a slide with the word "recall," which the patient responded to by stating aloud the word presented on the first slide of the trial. Training in each task occurred before operation, using different items.

Analysis. Each channel on the FM tape was digitized at $10 \mathrm{kHz}$ using a program running on a MacIntosh IIx computer. Activity of each microelectrode channel was divided into that of individual neurons using a window discriminator and visual separation of the resulting amplitudefrequency histograms. We have published previously examples of this separation (Schwartz et al., 1996). The time during which each slide was presented was divided into four temporal epochs. Epoch 1 was the time from slide drop to the opening of the projector shutter. Epoch 3 began $300 \mathrm{msec}$ before the beginning of the patient's overt response for those slides with overt responses or the average time for initiation of an overt response for slides without overt responses. This epoch lasted $1500 \mathrm{msec}$, encompassing activity related to output. Epoch 2 was the period from opening of the shutter to the beginning of epoch 3. Thus, epoch 2 included activity related to perception and processing. Epoch 4 was the period from the end of epoch 3 to the drop of the next slide. Normalized frequency of activity of each neuron was determined for each epoch of each slide by dividing the number of discharges in an epoch by the duration of that epoch.

Statistical analysis. The relationship to identification of names was established by comparing activity during each epoch of identification with that during the same epoch of the physically identical first slides of the perceptual control for names. The relationship to memory for names was established by comparing activity during each epoch of identification with that during the same epoch of the physically identical encoding (first) slide of the measure of recent memory for names. Similarly, the relationship to identification for words was established by comparing activity during each epoch of identification of words with that during the same epoch of the perceptual control, and the relationship to memory for words by comparing each epoch of identification of words with that during the physically identical encoding slides of the word memory measure. In addition to these primary comparisons establishing a relationship with identification or memory for words or names, activity during each epoch of the encoding slide of the memory measures was also compared with that during the same epoch of the perceptual control for that type of material.

Significance of each comparison was determined with nonparametric Mann-Whitney $U$ tests. The present study was designed to identify significant changes in any one epoch of these comparisons. Thus, significance was set at $p<0.005$. At this level, reporting of random significance in any one epoch or in the summed activity of all epochs has a two-tailed chance probability of 0.05 for each comparison. Because a neuron was related to identification or memory for words or names by a significant change in even one epoch or in overall activity in the primary comparison that establishes the relationship, each of those relationships has been determined with an overall two-tailed probability of 0.05 .

\section{RESULTS}

Technically satisfactory recordings during identification, memory, and perceptual control measures for names and words were obtained from 31 lateral temporal cortical neurons at 18 sites in the 12 patients, 7 neurons at 4 sites in 2 patients from the right nondominant temporal lobe, and the remainder from the left. Table 1 indicates where individual recordings were obtained in anterior middle temporal gyrus or the most lateral portion of inferior temporal gyrus, as well as the recording depth. Average activity of an individual neuron during all tasks was 3.73 discharges/sec (range of $0.24-12.65$ ), with maximal activity during any task of 17.2 discharges/sec.

\section{Overall changes in activity}

Twenty-seven of the 31 neurons had significant changes in at least one of the primary comparisons relating a neuron with identification or memory for words or names (Table 1). For 13 neurons, a significant change was present in only one comparison, six had significant changes on two comparisons, five had three significant comparisons, and three had significant changes on all four comparisons. However, only four neurons had significant changes in the same direction, excitation, or inhibition for both measures of the same function, either identification of both words and names or memory for both words or names. One additional neuron had significant changes in the same direction for both identification and memory for the same material: names.

\section{Changes with recent memory}

In the comparison used to establish a relationship with memory, physically identical material is presented with different instructions to identify aloud the name of the object or the word or to identify aloud and remember the explicit name or word. Thus, this comparison subtracts out activity associated with overt identification and leaves that associated with the instruction to retain the explicit item in recent memory. Significant differences in activity between these two conditions at $p<0.005$ were present for one or more epoch of 21 of the 31 neurons, including five of the seven neurons in right brain. Thirteen neurons had significant changes with encoding of memory for names, and 16 neurons had changes with memory for words. Significant changes in some epoch for both memory for names and words in the same direction were present for three neurons, for both but in opposite directions for five, and in only one memory measure for 13 . Both increases and decreases in activity were recorded, with 5 of 13 significant changes in memory for names increases, as were 9 of 16 significant changes in memory for words. Thus, neurons changing activity with memory were frequently encountered in this portion of temporal cortex and were widely distributed to both hemispheres, but only $14 \%$ of the neurons showed changes in the same direction during memory for both types of material.

The level of activity during the encoding phase of memory for names or words was also compared. This differed significantly $(p<0.005)$ during one or more epoch for seven neurons: a neuron that also had significant changes in the same direction for memory for both types of material compared with identification, two with opposite changes in memory for words or names on that comparison, three with changes in only one memory measure compared with identification of the same material, and one with no significant changes for either memory for words or names when compared with identification.

\section{Changes with identification}

In the comparison used to establish a relationship with identification of words or names, physically identical material was presented with the instruction to identify it aloud verbally (name or word reading) or to look at it silently, as a perceptual control. Seventeen neurons show significant changes $(p<0.005)$ in one or more epoch on this comparison, including three in the nondominant hemisphere. Thirteen neurons had significant changes with naming and 10 with word reading. Two neurons had significant changes in the same direction with both tasks; four had changes with both in the opposite direction, and 11 had changes with only 

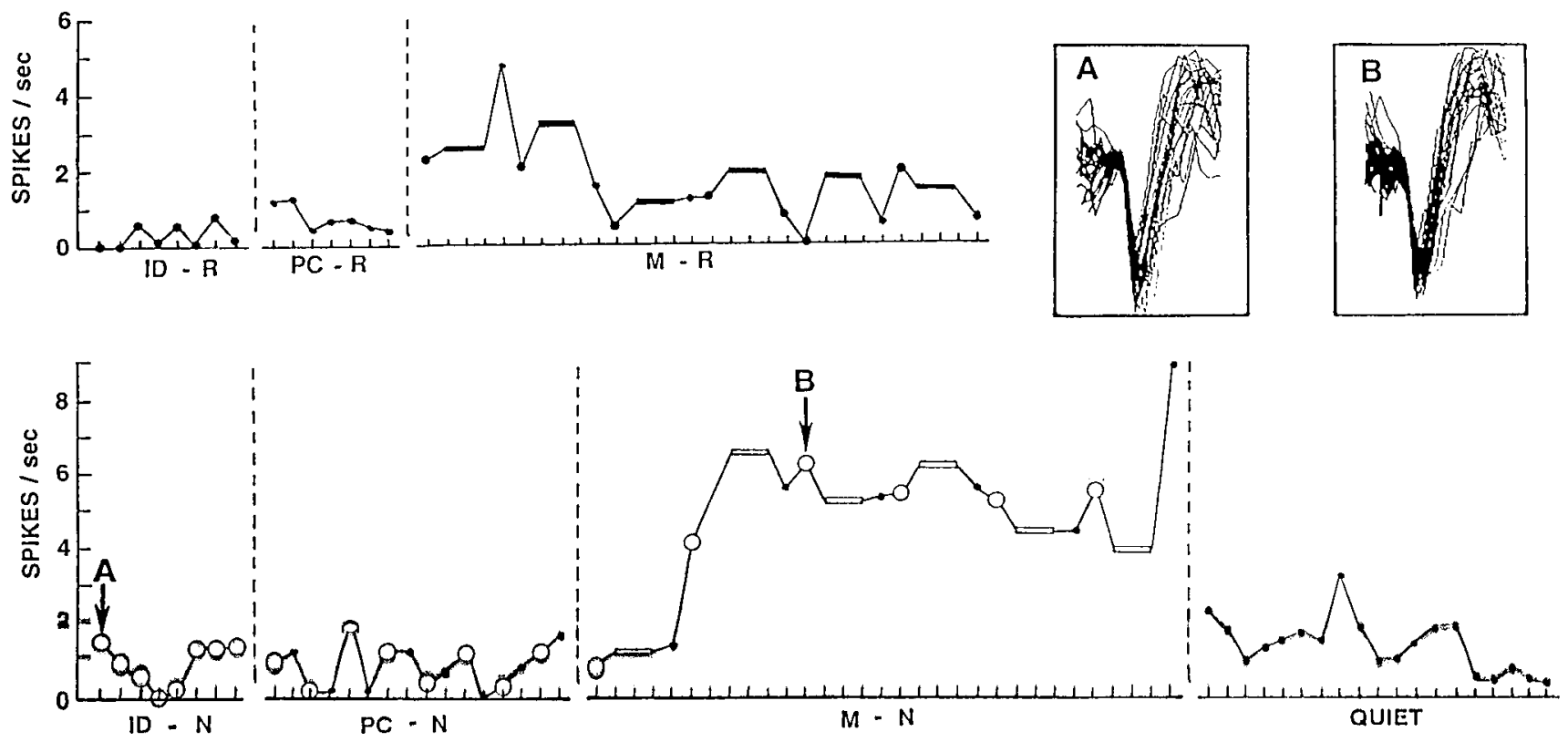

Figure 1. Frequency of activity of neuron $3 / 2$ from case 4 (Table 1 ) during each trial of identification of namable objects $(I D-N)$ and words $(I D-R)$, recent memory for object names $(M-N)$ and words $(M-R)$, the perceptual controls for names $(P C-N)$ and words $(P C-R)$, and a quiet period that concluded the recordings. Activity is averaged for all epochs of each item, and that for the three distracting items in each trial of the recent memory measures is averaged together (bars). The sets of items identified by the large circles for identification of names, the perceptual control, and the encoding phase of the recent memory measure for names include physically identical stimuli that, for identification and memory encoding, elicited the same overt responses. The insets show superimposed traces of 20 consecutive action potentials recorded during the first item of identification of names $(A)$ and the encoding phase of the third trial of recent memory for names $(B)$. Each mark on the $x$-axis is 4 sec, the period each item was shown. Each task was separated by a brief period of review of the instructions for the following task. Activity during those instructions was not assessed. The order of the tasks for this case is as shown, beginning with word identification and ending with the quiet period. Note the sustained increase in activity with memory for names, which disappears in the following quiet period and is evident to a much lesser degree with memory for words, and not at all for identification or perceptual controls for words or names. Activity throughout this recording appears to be from the same neuron, as indicated by the insets, in which action potential configuration is the same whether recorded during a low level of activity with name identification or a high sustained level of activity during memory for names.

naming or reading. Five of the 13 changes with naming were increases, as were 5 of the 10 with reading. Seventeen neurons had one or more epoch with significantly different levels of activity during naming compared with reading, including 11 that also had significant changes from the perceptual control for naming or reading. Nine neurons had greater activity with naming. Neurons with significant changes with identification were also rather often encountered, although changes were usually for only naming or reading and not both.

\section{Relationship between changes with identification and memory for the same material}

Eighteen neurons had significant changes in one or more epoch with identification, the encoding phase of the memory measure for names, or both. Twelve of these neurons had changes in the opposite direction between naming and memory for those names (seven) or changes only with memory (five). One neuron showed significant changes in the same direction (decreases) during both identification and memory, with a greater reduction in activity with memory than with identification. Five other neurons had changes only with identification. Only in those neurons was activity not statistically distinguishable between identification and the encoding phase of memory for names.

Twenty-one neurons had significant changes in one or more epoch during identification, memory for words, or both. Sixteen of these neurons had changes in the opposite direction between word identification or memory for those words (five) or only with memory (11). Five neurons had changes with word identification and no significant difference with word memory; none had significant changes in the same direction for both.

Thus, for most neurons, regardless of the type of material, the instruction to retain an item in memory often changes the frequency of activity from that during identification of the same material. This effect involves the disappearance of a change that occurs with identification, with activity during memory encoding returning to the level of the perceptual control, or a further change in the opposite direction. To determine that, activity during the encoding phase of memory was compared with the perceptual control for the same material. Of the 23 primary identification-perceptual control comparisons that related a neuron with identification of names or words, only six had significant changes in the same direction when the encoding phase of memory for the same type of material was compared with the appropriate perceptual control (Table 1), although the name or word is spoken aloud in both identification and memory encoding. On the other hand, of the 16 sets of comparisons that related a neuron only with memory for words or names based on the primary identification-memory comparison, 11 showed significant change in the same direction on the comparison between memory encoding and the appropriate perceptual control. Thus, when compared with a common perceptual control, in $74 \%$ of neurons with changes related to identification, these disappeared with the instruction to retain items in memory, but changes in neurons related to memory alone persisted, regardless of whether the 

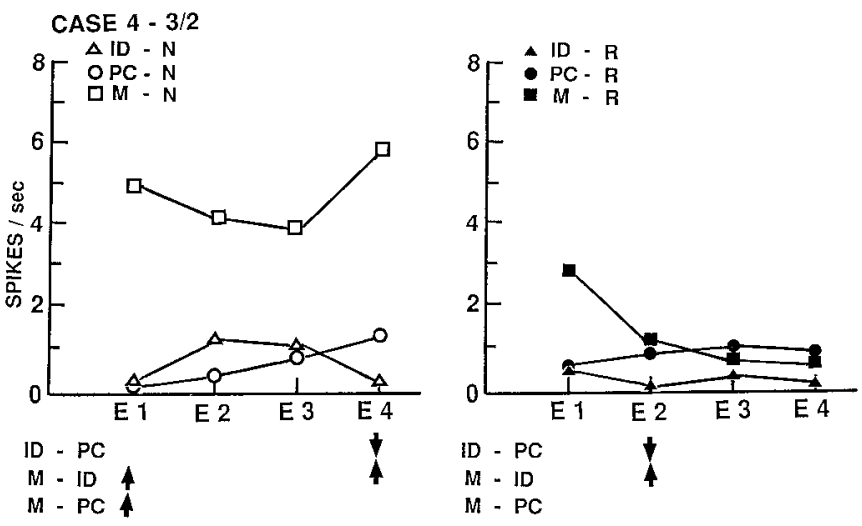

Figure 2. Activity from the same neuron as Figure 1 (case 4, neuron 3/2) but averaged by temporal epoch (epochs defined in Materials and Methods) for all trials of each task: identification of names $(I D-N)$ or words $(I D-R)$, encoding phase of memory for names $(M-N)$ or words $(M-R)$, and perceptual controls for names $(P C-N)$ or words $(P C-R)$. Statistically significant changes $(p<0.005)$ are indicated below for identificationperceptual $(I D-P C)$, memory-identification $(M-I D)$, and memory-perceptual control $(M-P C)$ comparisons, with an up arrow indicating a significant increase in that epoch for that comparison and a down arrow indicating a significant decrease. Superimposed on the sustained change illustrated in Figure 1 are some phasic changes related to presentation and response to the items: decreases for the encoding phase of memory for names and for identification of words, and increases for identification of names.

comparison was with identification or with the perceptual control for that type of material.

\section{Changes within memory tasks}

The memory tasks include encoding, storage, and retrieval phases. Changes in activity between these phases were compared for memory for names or words. Only one neuron (case 2, 1/1) showed significant differences between phases of the memory tests, with significantly decreased activity during retrieval of words or names compared with activity during encoding or storage.

\section{Nature of changes in activity}

The left temporal neuron with activity illustrated in Figures 1 and 2 (case 4,3/2) demonstrates the several types of changes in activity observed in this study. In Figure 1, activity is recorded for each trial of the four tasks and perceptual controls. Aside from a small increase on the first two trials of memory for words, activity is quite stable until an abrupt increase appears with the first trial of the name memory task. This increase is sustained for the $2 \mathrm{~min}$ of that task and then disappears in the quiet period that follows. The recordings during all tasks are clearly from the same single neuron because action potential configurations are identical, as illustrated for name identification and memory for names. The patient produced overt outputs on every trial except those of the perceptual control and quiet periods. Thus, producing an overt output does not account for the appearance of the abrupt and sustained change with name memory. The name memory task is no more demanding than the word memory task, and both have the same format, so task difficulty would not account for the change. Physically identical stimuli were used for the circled trials of name identification, perceptual control, and encoding phases of the name memory task, so the nature of the material also will not account for the change.

In Figure 2, the same activity is averaged by temporal epochs.
A CASE $10-4 / 1$
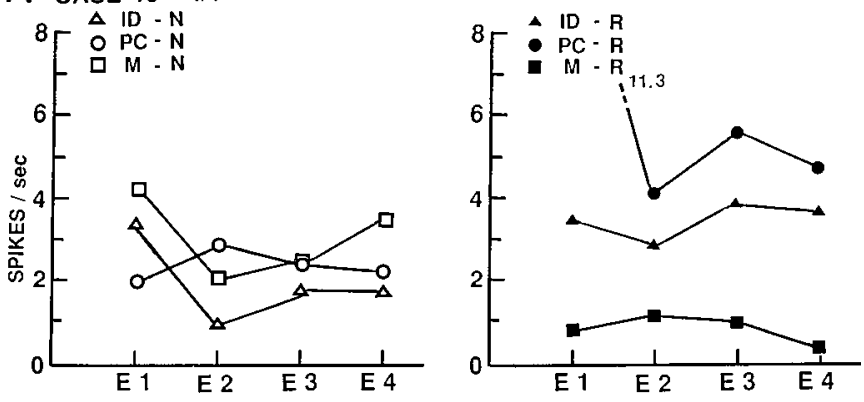

ID - PC

$M-$ ID

$M-P C$

ID - PC

$M-I D$
$M-P C$

B CASE $10-4 / 2$
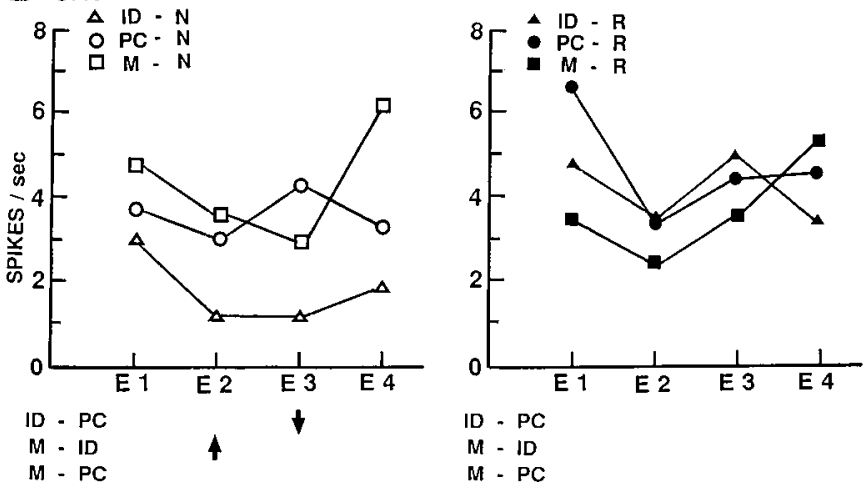

Figure 3. Activity of two neurons recorded simultaneously by electrode 4 in left middle temporal gyrus of case 10, averaged by temporal epochs. Format of illustration and abbreviations as in Figure 2. The neuron shown in $A$ has a significant sustained decrease in activity during the encoding phase of recent memory for words in epoch 3 and overall activity compared with word identification, and epochs 1,3 , and 4 and overall activity compared with the perceptual control for words but no changes with name tasks. The nearby neuron shown in $B$ has transient changes in epochs 2 and 3 during name identification that disappears with the instruction to retain those names in recent memory, with no changes with word tasks.

Superimposed on the sustained change is a small transient one, with increased activity in epochs 2 and 3 of name identification and decreased activity in the same epochs with name memory encoding. The result of this "mirror image" transient change is that the statistically significant differences are confined to epochs 1 and 4 for the name memory increase (compared with either name identification or the perceptual control), and to epoch 4 for the decrease for name identification (compared with the perceptual control). The significant change for word identification is a reduction in epoch 2 that disappears in the encoding phase of memory for words. Although this particular neuron is the only one with significant changes in the same direction for identification for both types of material (decreases with both) and for memory for both types of material (increases with both), the nature of the changes in activity for the two types of material is different. Moreover, this neuron shows both transient changes and a sustained change.

Figures 3-5 depict activity of four additional neurons from three other patients, also averaged by temporal epochs. The neuron of Figure $3 A$ has a sustained decrease in activity during memory for words, with no other statistically significant changes. A nearby neuron recorded simultaneously through the same microelectrode (Fig. 3B) shows no changes with word tasks but a 


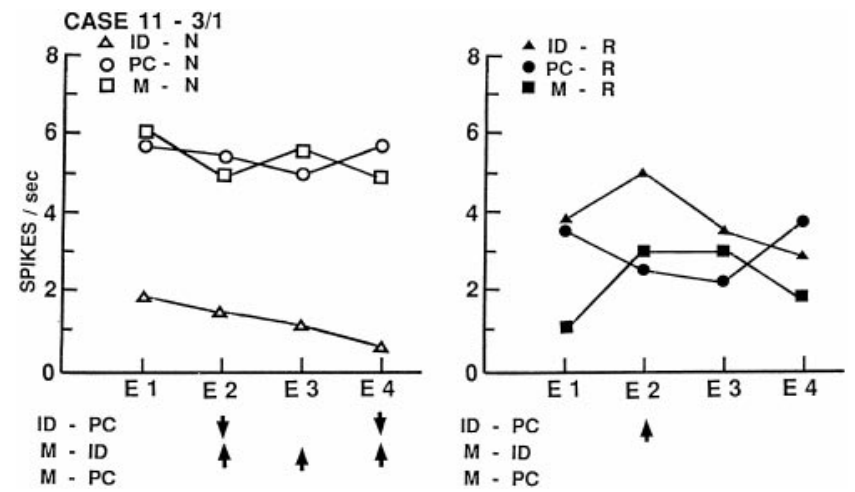

Figure 4. Activity of neuron 3/1 from left inferior temporal gyrus of case 11, averaged by temporal epochs and presented as in Figure 2. There is a sustained decrease in activity during name identification with significant changes in epochs 2 and 4 and overall activity, which disappears with the instruction to retain those names in recent memory with significant changes in epochs 2,3, and 4 and overall activity. During word tasks, there is a significant transient increase in word identification, epoch 2, which disappears with word memory as overall activity is significantly decreased from that during word identification.
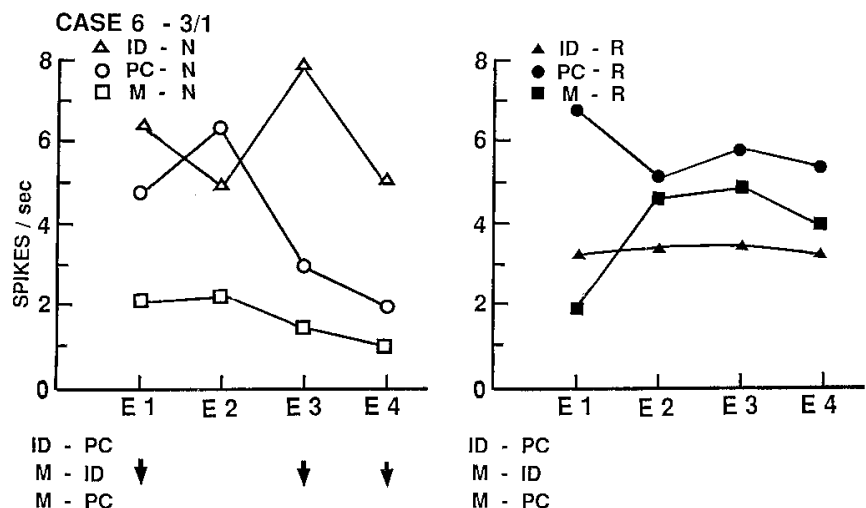

ID - PC

$M-I D$

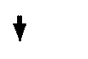

$\downarrow$

$M-P C$

Figure 5. Activity of neuron 3/1 from left middle temporal gyrus of case 6 , averaged by temporal epochs and presented as in Figure 2. There is a sustained decrease during the encoding phase of recent memory for names with significant changes in epochs 1,3 , and 4 and in overall activity and no changes during name identification or word tasks.

transient decrease in activity during name identification that disappears during the encoding phase of memory for those names. The neuron of Figure 4 shows a sustained decrease in activity with name identification that disappears with memory for those names and a transient increase with word identification that also disappears with memory for those words. The neuron of Figure 5 shows only a sustained decrease during memory for names. These neurons show changes in the same direction to only one of the tasks sampled here. Changes with identification disappear during the encoding phase of memory for that material, although the conditions differ only in the instruction to retain the item in recent explicit memory. Individual neurons show both sustained and transient changes during different tasks.

Sustained changes were most commonly identified. The 31 neurons have 52 significant comparisons between identification and the perceptual control or memory and identification, during one or more epoch. Only 16 of these (31\%) involve only epochs 2,3 , or both and are thus likely transient changes (Table 1; Figs. 2 , word, $3 B, 4$, word). These transient changes were recorded somewhat more frequently during the word identification task than the other tasks. The remaining significant comparisons involve overall activity, the first and last epochs, or three or more epochs, all of which have been related to sustained activity (Figs. 2, name, $3 A$, 4, name, 5).

\section{Activity in nearby neurons}

There are ten sets of two or three nearby neurons so close together that each set was recorded simultaneously through the same microelectrode (Table 1). Four of these sets have no significant changes in common between the nearby neurons (Fig. 3). Two other sets of three nearby neurons have changes in one function common to two neurons (although in one set in opposite directions) and no changes common to all three. The remaining four sets had one function with changes common to all the recorded nearby neurons; in three, the common changes were on one memory measure. However, in one set, the common memory changes were not all in the same direction. Only one set had identical changes in both nearby neurons. In the remaining sets with changes in one function in common, there were other changes present in only some of the nearby neurons. Simultaneous recordings were obtained at two different cortical depths in two patients (case 2, electrodes 3 and 4; case 10, electrodes 3 and 4). No changes in the same direction occur at the two levels; for word memory, one set of recordings shows opposite changes at the two depths.

\section{DISCUSSION}

In the temporal cortex sampled in this study, statistically significant changes in neuronal activity indicating participation in the networks for identification or recent memory for words or object names were frequently encountered in recordings from either hemisphere. Neurons in each of these networks are widely distributed. This finding is similar to those in our earlier studies of changes in human cortical neuronal activity with language and memory. Neurons changing activity with word reading or naming (compared with perceptual controls) were present in equal proportions in both dominant and nondominant temporal lobes (Weber and Ojemann, 1995; Schwartz et al., 1996). Similarly, neurons changing activity with recent memory for words (compared with word identification) were equally distributed in temporal cortices of both hemispheres (Weber and Ojemann, 1995), as were neurons changing activity with auditory word repetition (compared with a tone) (Creutzfeldt et al., 1989). The present study includes the first recordings from nondominant hemisphere during a measure of recent memory for names, with the same finding of changes in activity in nondominant, as well as dominant, temporal cortex. In the present study, there is no suggestion of a difference in direction of changes, inhibition, or excitation between the hemispheres, although, in an earlier study of changes with naming, inhibition was significantly more frequent in recordings from the dominant hemisphere (Schwartz et al., 1996).

This lack of lateralization of neuronal activity contrasts with the known lateralization of essential areas for these behaviors, defined as those areas in which damage leads to a deficit. Within the dominant hemisphere, essential areas for the identification tasks are localized to focal areas outside the region from which the recordings reported here were obtained (Ojemann, 1983; Ojemann et al., 1989a). Thus, substantial portions of the widespread neural networks subserving those functions, including those portions from which our recordings were obtained, have sufficient redundancy so that excision of that portion of the network does not result in a measurable deficit. 
Although neurons changing activity with recent memory for words or names were widely distributed, any individual neuron seldom changed activity in the same way for both. Moreover, some of the few neurons with changes in the same direction for both had quite different patterns of activity with memory for the different material, as shown in Figures 1 and 2. The cortical networks subserving recent memory for different types of verbal material are primarily separate. In the present study, any individual neuron also seldom changed activity in the same way during identification of words or names. A similar separation of neurons changing activity with these two tasks was reported by Schwartz et al. (1996) in recordings from other patients.

The introduction of the instruction to retain an explicit item in recent memory often changes the level of activity compared with that during identification of those items, even when the actual task performed is identical, as when an item is named aloud in both the identification task and the encoding phase of the memory task. This effect ranges from a loss of changes present with identification compared with a perceptual control, to significantly greater activity in the opposite direction, beyond that present with the perceptual control. Indeed, in only $\sim 25 \%$ of the neurons related to identification was the same change still present during memory encoding. The neural substrate for explicit memory for an item differs substantially from the neural substrate for generalized semantic memory for the same item. Separation of cortical areas essential for naming from those essential for recent explicit memory for the same names has also been identified in electrical stimulation mapping studies (Ojemann, 1978, 1983; Ojemann and Dodrill, 1985). Because there is often different activity during explicit memory compared with identification of the same item, as well as different activity for words compared with names, most neurons had significant changes in one direction for only one of the behaviors sampled in this study. There is little evidence of multipurpose neurons changing activity in the same direction with multiple behaviors.

In the present study, reversal of activity with the instruction to retain an item in memory involved disappearance of both excitatory and inhibitory changes present with identification. In a separate study, anterior temporal neurons with reductions in activity during identification of words (compared with a perceptual control) that reversed on the encoding phase of recent memory for words were found to also have significant changes with associative word learning, with greater activity for word pair associations learned rapidly compared with those learned slowly or not at all (Ojemann and Schoenfield-McNeill, 1998). Neurons with other types of changes during identification or memory did not show this effect during associative learning. Thus, the neurons in the present study with inhibition during identification that reverses with recent memory encoding (Figs. 2, 3B, 4, names) are likely primarily part of networks establishing associations to that verbal material.

The level of activity of neurons recorded in the present study was relatively low, $1-3 \mathrm{~Hz}$ for many control measures, with maximum increases to $17 \mathrm{~Hz}$. These levels are similar to those observed in other human temporal cortical recordings (Ojemann et al., 1988; Creutzfeldt et al., 1989; Haglund et al., 1994; Schwartz et al., 1996) and some nonhuman primate inferior temporal gyrus recordings (Fuster and Jervey, 1982). The present study was designed so that significant changes could be identified in individual temporal epochs, as well as in overall activity. Slightly less than one-third of comparisons with significant changes in any epoch had transient changes, in epochs 2 and 3, the ones associated with processing and output. Most of the remaining changes were task-specific sustained shifts in overall level of activity. These sustained shifts lasted minutes, during the duration of the specific task (Fig. 1). They do not appear to be artifactual as might occur with electrode movement, for they appeared with a specific task and disappeared on its completion, and action potential configurations remained stable throughout the recording. Task-specific sustained changes have been identified in other human temporal neuronal recordings. A sustained reduction in activity was observed in an anterior temporal neuron recorded during overt and silent naming in one language (English) and not another (Spanish), with an abrupt transition with the instruction to name in a different language and the abrupt reappearance of the sustained decrease during a second period of naming in English (Ojemann, 1990). Sustained excitation throughout overt oral naming and not silent or sign naming of the same objects was identified in an anterior temporal recording from another subject (Haglund et al., 1993). In those lateral temporal cortical neurons in which encoding activity differentiated between word pair associations that a subject learned rapidly or poorly, the increased activity for rapidly learned pairs was sustained seconds longer than activity for poorly learned pairs (Ojemann and Schoenfield-McNeill, 1998). Both sustained increases and decreases have been observed. However, as demonstrated in the present study, neurons are not "tonic" or "phasic" for an individual neuron may show transient phasic changes for one behavior, and sustained tonic or mixed sustained and transient changes with other behaviors, as illustrated in Figures 1, 2, and 4. A prominent component of the temporal cortical neuronal activity during language or recent verbal memory tasks, then, are these long-lasting task-specific sustained shifts in level of activity, sometimes with superimposed transient modulations temporally related to the task.

These task-specific sustained shifts seem most compatible with a model in which neurons convey information in the network subserving the specific function by average action potential frequency rather than patterns of activity, a conclusion also reached in studies of activity during recognition memory in inferior temporal cortex of nonhuman primates (Miller and Desimone, 1993) and on more general theoretical grounds (Shadlen and Newsome, 1998). Sustained changes have been related to different levels of attention. However, the level of attention demanded by the memory for words task in our human study would seem to be the same as that for the memory for names task, although only one showed a sustained change in the activity of the neurons of Figure 1, 3A, and 5. One hypothesis for the mechanism behind these sustained changes is activity of thalamocortical activating circuits with the specific behavior. Activity of these circuits has been proposed as the basis for changes in memory for names evoked by human ventrolateral thalamic stimulation (Ojemann, 1976). Activity of thalamocortical activating circuits has also been proposed as the basis for the electrocorticographic change, local desynchronization, which appears during naming localized to human temporal cortical sites independently identified as essential for naming by electrical stimulation mapping (Ojemann et al., 1989b).

Nearby neurons, close enough to be recorded through the same microelectrode, seldom showed qualitatively similar changes for the same behavior. This is similar to our findings in several previous studies in which nearby neurons recorded during a variety of behaviors were usually related to different functions (Ojemann et al., 1992; Schwartz et al., 1996), although another of our studies found a high proportion of nearby neurons changing 
activity with memory for names (Haglund et al., 1994). The pattern of nearby neurons related to different behaviors is not the finding expected if the portion of human temporal association cortex sampled in the present study is organized in columns each related to an individual function. It seems more compatible with a model in which networks of neurons related to different functions are interlaced. Whether the same interlacing cortical networks may repeat at different depths in the same cortical "column" is uncertain because there is not yet a sufficient number of successful recordings at two cortical depths. The two examples in the present study seem to relate neurons at the different depths to different functions.

Although all the recordings reported here were from patients with epilepsy, it is unlikely that the observed changes in neuronal activity reflect local effects of that disease. The surgical approach used in these patients, a standard one for temporal lobe epilepsy (Spencer et al., 1984; Ojemann, 1995), includes resection of anterior portions of middle and inferior temporal gyri to provide access to the epileptogenic zone in medial temporal lobe. Thus, the sites from which our recordings were obtained are not the primary source of these patients' seizures and are little involved in the epileptic process. Electrocorticographic recording from those structures rarely showed epileptiform activity. Individual neuronal recordings did not show the bursting activity associated with epilepsy (Calvin et al., 1973). Histological examination of this tissue showed normal cortical architecture. Unfortunately, there is no technique for obtaining human neuronal recordings other than in neurosurgical settings, which inevitably are in special patient populations. Nevertheless, the findings from these special populations provide hypotheses about the organization of the neural networks subserving uniquely human functions whose generalized applicability can be assessed by testing them in other populations as techniques for such recordings become available.

Activity recorded from inferior temporal cortex of nonhuman primates during memory measures has shown some, but not all, of the major features identified here. Changes specific to identification or memory for one class of items or individual items have been rather frequently identified in nonhuman recordings (Fuster and Jervey, 1982; Brown et al., 1987; Miller et al., 1991; Miller and Desimone, 1993). Sustained changes have also been reported (Miller and Desimone 1993; Fuster and Jervey, 1982), although these changes were sustained throughout the presentation of specific items in some studies (Brown et al., 1987; Miller and Desimone, 1993) or throughout the task but at lower levels during storage compared with encoding and retrieval (Fuster and Jervey, 1982). Other studies have not identified sustained activity (Baylis and Rolls, 1987).

Comparison of activity during identification and when the same item is retrieved from memory has shown no differences in some nonhuman primate recordings (Fuster and Jervey, 1982), although others have reported differences, often less activity when the item is retrieved from memory (Baylis and Rolls, 1987; Brown et al., 1987; Miller et al., 1991; Miller and Desimone, 1993). The nonhuman studies all use visuospatial material in various delayed match-to-sample recognition paradigms compared with the verbal material with cued recall used in our human studies. The limited experience with human lateral temporal recordings during memory for visuospatial material indicates a predominance of inhibitory processes, with a higher level of activity when the same material was directly matched compared with encoding or retrieval from explicit memory in a delayed match paradigm (Holmes et al., 1996). Overall, both human and nonhuman primate studies indicate separation of neural networks for different material, with some evidence from nonhuman studies supporting the human findings of different levels of activity with retention of an item in recent explicit memory compared with its identification and of sustained activity as one feature of the networks subserving these functions.

\section{REFERENCES}

Baylis GC, Rolls ET (1987) Responses of neurons in the inferior temporal cortex in short term and serial recognition memory tasks. Exp Brain Res 65:614-622.

Benton A, Hannay H, Varney N (1975) Visual perception of line direction in patients with unilateral brain disease. Neurology 25:907-910.

Brown MW, Wilson FAW, Riches IP (1987) Neuronal evidence that inferomedial temporal cortex is more important than hippocampus in certain processes underlying recognition memory. Brain Res 123:241-259.

Calvin W, Ojemann G, Ward Jr A (1973) Human cortical neurons in epileptogenic foci: comparison of interictal firing patterns to those of "epileptic" neurons in animals. Electroencephalogr Clin Neurophysiol 34:337-351.

Creutzfeldt O, Ojemann GA, Lettich E (1989) Neuronal activity in the human lateral temporal lobe. I. Responses to speech. Exp Brain Res $77: 451-475$.

Fried I, Mateer C, Ojemann G, Wohns R, Fedio P (1982) Organization of visuospatial functions in human cortex: evidence from electrical stimulation. Brain 105:349-371.

Fuster JM, Jervey JP (1982) Neuronal firing in the infertemporal cortex of the monkey in a visual memory task. J Neurosci 2:361-375.

Haglund MM, Ojemann GA, Lettich E, Bellugi U, Corina D (1993) Dissociation of cortical and single unit activity in spoken and signed language. Brain Lang 44:19-27.

Haglund MM, Ojemann GA, Schwartz TW, Lettich E (1994) Neuronal activity in human lateral temporal cortex during serial retrieval from short-term memory. J Neurosci 14:1507-1515.

Holmes MD, Ojemann GA, Lettich E (1996) Neuronal activity in human right lateral temporal cortex related to visuospatial memory and perception. Brain Res 711:44-49.

Miller E, Desimone R (1993) Activity of neurons in anterior inferior temporal cortex during a short-term memory task. J Neurosci 13:1460-1478.

Miller E, Li L, Desimone R (1991) A neural mechanism for working and recognition memory in inferior temporal cortex. Science 254: 1377-1379.

Milner B (1967) Basic mechanisms suggested by studies of temporal lobes. In: Basic mechanisms underlying speech and language. (Millikan C, Darley F, eds), pp 122-131. New York: Grune and Stratton.

Milner B (1971) Disorders of learning and memory after temporal lobe excision in man. Clin Neurosurg 19:421-446.

Ojemann G (1976) Subcortical language mechanisms. Stud Neurolinguistics 1:103-108.

Ojemann G (1978) Organization of short-term verbal memory in language areas of human cortex: evidence from electrical stimulation. Brain Lang 5:331-348.

Ojemann G (1983) Brain organization for language from the perspective of electrical stimulation mapping. Behav Brain Sci 6:189-206.

Ojemann G (1990) Organization of language cortex derived from investigations during neurosurgery. Semin Neurosci 2:297-305.

Ojemann G (1995) Awake operations with mapping in epilepsy. In: Operative neurosurgical techniques, Ed 3 (Schmidek HH, Sweet WH, eds), pp 1317-1322. Philadelphia: Saunders.

Ojemann G, Dodrill C (1985) Verbal memory deficits after left temporal lobectomy for epilepsy: mechanisms and intraoperative prediction. J Neurosurg 62:101-107.

Ojemann G, Dodrill C (1987) Intraoperative techniques for reducing language and memory deficits with left temporal lobectomy. Adv Epileptol 16:327-330.

Ojemann G, Schoenfield-McNeill J (1997) How often do individual neurons in human temporal cortex show qualitatively similar activity during different verbal behaviors? Soc Neurosci Abstr 23:1059.

Ojemann G, Schoenfield-McNeill J (1998) Neurons in human temporal cortex active with associative learning. Brain Lang 64:317-327.

Ojemann G, Creutzfeldt O, Lettich E, Haglund MM (1988) Neuronal 
activity in human lateral temporal cortex related to short-term verbal memory, naming and reading. Brain 111:1383-1403.

Ojemann G, Ojemann J, Lettich E, Berger M (1989a) Cortical language localization in left, dominant hemisphere: an electrical stimulation mapping investigation in 117 patients. J Neurosurg 71:316-326.

Ojemann G, Fried I, Lettich E (1989b) Electrocorticographic (ECoG) correlates of language. I. Desynchronization in temporal language cortex during object naming. Electroencephalogr Clin Neurophysiol 73:453-463.

Ojemann G, Ojemann S, Fried I (1998) Lessons from the human brain: Neuronal activity related to cognition. The Neuroscientist 4:285-300.

Ojemann JG, Ojemann GA, Lettich E (1992) Neuronal activity related to faces and matching in human right nondominant temporal cortex. Brain 115:1-13.

Perrine K, Devinsky O, Uysal S, Luciano D, Dogali M (1994) Left temporal neocortex mediation of verbal memory. Neurology 44:1845-1850.

Schwartz TH, Ojemann GA, Haglund MM, Lettich E (1996) Cerebral lateralization of neuronal activity during naming, reading, and linematching. Cognit Brain Res 4:263-273.
Scoville W, Milner B (1957) Loss of recent memory after bilateral hippocampal lesions. J Neurol Neurosurg Psychiatry 20:11-21.

Shadlen M, Newsome W (1998) The variable discharge of cortical neurons: implications for connectivity, computation and information coding. J Neurosci 18:3870-3896.

Shimamura A (1989) Disorders of memory: the cognitive science perspective. In: Handbook of neuropsychology (Boller F, Grafman J, eds), pp 35-74. Amsterdam: Elsevier.

Spencer D, Spencer S, Mattson R, Williamson P, Novelly R (1984) Access to posterior medial temporal lobe structures in the surgical treatment of temporal lobe epilepsy. Neurosurgery 15:667-671.

Squire L, Zola-Morgan M (1991) The medial temporal lobe memory system. Science 253:1380-1386.

Wada J, Rasmussen T (1960) Intracarotid injections of sodium amytal for the lateralization of cerebral speech dominance. J Neurosurg 17:266-282.

Weber PB, Ojemann GA (1995) Neuronal recordings in human lateral temporal lobe during verbal paired associate learning. NeuroReport 6:685-689. 